Supporting Information for:

\title{
Fundamental limits to the electrochemical impedance stability of dielectric elastomers in bioelectronics
}

Paul Le Floch ${ }^{1}$, Nicola Molinari ${ }^{1}$, Kewang Nan ${ }^{1}$, Shuwen Zhang ${ }^{1}$, Boris Kozinsky ${ }^{1}$, Zhigang $\mathrm{Suo}^{1,2}, \mathrm{Jia} \mathrm{Liu}^{1 *}$

${ }^{1}$ John A. Paulson School of Engineering and Applied Sciences, Harvard University, Cambridge, MA 02138, USA

${ }^{2}$ Kavli Institute for Bionano Science and Technology, Harvard University, MA 02138

*Corresponding author: Email: jia_liu@ seas.harvard.edu, Phone: 617-496-8119

\section{This PDF file includes:}

Materials and Methods

Supplementary Notes

Figures S1-S10 


\section{Materials and Methods}

\section{Preparation of thin film dielectrics encapsulating electrodes.}

\section{Shadow mask preparation}

1. Design on CoreDraw.

2. Cut the shape in a 2 mil-thick film of PVC using a VeraLaser Cutting/Engraving System.

3. Rinse the mask with Isopropyl Alcohol (IPA), de-ionized (DI) Water and then blow dry.

4. Put the mask in a vacuum chamber overnight.

\section{Gold electrode deposition}

1. Rinse glass slide or silicon oxide wafer with acetone, then IPA, then DI Water and blow dry.

2. Attach the shadow mask to the substrate using double-sided Kapton tape.

3. Deposition of $5 \mathrm{~nm}$ of Chromium (or Titanium) as an adhesion layer, then $100 \mathrm{~nm}$ of Gold using an E-Beam evaporator (Sharon Vacuum Co., Inc.).

4. Remove the shadow mask.

5. Blow dry to remove any particle before spin coating of any material.

\section{Silver nanoparticles - polymer electrode preparation}

1. Prepare electrode Gerber design file using KiCAD.

2. Rinse glass slide or silicon oxide wafer with acetone, then IPA, then DI Water and blow dry.

3. Use a Voltera V-One PCB printer with Dupont PE873 silver-based ink to print the design on the glass slide.

4. Bake the ink at $160{ }^{\circ} \mathrm{C}$ for 20 minutes, with a 5 minutes rise time.

\section{Carbon nanotubes electrode preparation}

1. Dissolve P3-SWNT (from Carbon Solution, Inc.) in DI water following the procedure from ${ }^{1}$.

2. Mix $3 \mathrm{~mL}$ of the solution to $12 \mathrm{~mL}$ of IPA.

3. Sonicate for $30 \mathrm{~s}$.

4. Filter the solution on a PTFE filter (Advantec T020A047A Unsupported Hydrophobic PTFE Membranes, $0.2 \mu \mathrm{m}, 47 \mathrm{~mm}$ diameter).

5. Transfer the CNT electrode on an elastomer substrate using a shadow mask.

\section{Thin film dielectrics preparation}

Different dielectric materials were spin coated on top of electrodes prepared in Section I for testing. Due to the polymer properties, the size for SEBS samples is set as $1 \mathrm{~cm}^{2}$ and sizes for all the other polymer samples are set as $4 \mathrm{~cm}^{2}$ to yield high-quality and defect free film while maintaining interfacial impedance values in the range of detection.

\section{SU-8 2002 bi-layer preparation}

1. Spin coat SU-8 2002: (1) Ramp to $500 \mathrm{rpm}$ at $100 \mathrm{rpm} / \mathrm{s}$ in $5 \mathrm{~s}$; (2) Ramp to $2500 \mathrm{rpm}$ at $300 \mathrm{rpm} / \mathrm{s}$ and then hold for $40 \mathrm{~s}$.

2. Bake at $65^{\circ} \mathrm{C}$ for $1 \mathrm{~min}+95^{\circ} \mathrm{C}$ for 3 minutes. 
3. UV $23 \mathrm{~s}$ at $6.67 \mathrm{~mW} / \mathrm{cm}^{2}$ (for a total exposure larger than $150 \mathrm{~mJ} / \mathrm{m}^{2}$ ), to avoid pinholes for a flat layer.

4. Cure at $65^{\circ} \mathrm{C}$ for $1 \mathrm{~min}+95^{\circ} \mathrm{C}$ for $50 \mathrm{~s}$.

5. Develop (SU-8 developer for $90 \mathrm{~s}$, wash each pattern closely with fresh SU8 developer for $15 \mathrm{~s}$ ). The only pattern to develop in this case is the tip of the electrode interconnect, so it can be clamped to the working electrode of the electrochemical impedance spectroscopy system.

6. Rinse with IPA and blow dry.

7. Bake at $65{ }^{\circ} \mathrm{C}$ for 1 minutes $95{ }^{\circ} \mathrm{C}$ for 1 minutes $140{ }^{\circ} \mathrm{C}$ for 5 minutes (ramping) $180{ }^{\circ} \mathrm{C}$ for 5 minutes (ramping) $200{ }^{\circ} \mathrm{C}$ for 20 minutes (ramping). Then ramp down to ambient temperature.

8. Repeat this procedure a second time to avoid pinholes.

\section{PDMS 10:1 (Sylgard 184) preparation}

1. PDMS base and curing agent are mixed for two minutes and degassed for two minutes using a THINKY MIXER (non-vacuum type).

2. Spin coat PDMS on the electrode in two steps. A rise to $500 \mathrm{rpm}$ at $100 \mathrm{rpm} / \mathrm{s}$ with a 5 seconds plateau, followed by a step in the range 5000-8000 rpm with a rise at $500 \mathrm{rpm} / \mathrm{s}$ and a hold of $60 \mathrm{~s}$.

3. Bake at $65^{\circ} \mathrm{C}$ overnight.

4. For the encapsulation of Carbon nanotube electrodes, a first layer of PDMS was spin coated and baked. Since nanotubes tend to diffuse and swell into PDMS, a second layer of PDMS was spin coated on top of it to ensure full encapsulation of the stretchable conductor.

\section{SEBS (Tuftec ${ }^{\mathrm{TM}} \mathrm{H} 1062$ preparation)}

1. Dissolve 70 to $100 \mathrm{mg} / \mathrm{mL}$ of SEBS in Toluene. Stirring for 48 hours at $40{ }^{\circ} \mathrm{C}$ in a closed container

2. Spin coat the solution immediately after pouring it on the electrode. The spin coating procedure is one step in the range $1000-2000 \mathrm{rpm}$ for $60 \mathrm{~s}$. The rise lasts $3 \mathrm{~s}$.

3. Dry at $80{ }^{\circ} \mathrm{C}$ overnight.

4. For the encapsulation of Silver nanoparticles - polymer electrodes, a first layer of SEBS was spin coated and baked. Since nanoparticles could diffuse and swell into SEBS, a second layer of SEBS was spin coated on top of it to ensure full encapsulation of the stretchable conductor.

\section{PIB (Exxon TM Butyl 268S) preparation}

1. Dissolve Butyl 268S (provided Vanderbilt Chemicals, LLC) in Toluene at $10 \%$ of solvent weight.

2. Spin coat the solution immediately after pouring it on the electrode. The spin coating procedure is one step in the range $1000-2000 \mathrm{rpm}$ for $60 \mathrm{~s}$. The rise lasts $3 \mathrm{~s}$.

3. Dry at $80^{\circ} \mathrm{C}$ overnight.

\section{Thickness measurements}


All the thickness measurements have been carried out using a Bruker Dektak Xt Stylus profiler. The force applied was set to 3 milligrams, and the scan speed to $0.67 \mu \mathrm{m} \cdot \mathrm{s}^{-1}$. Two-points surface leveling was applied using the commercial software of the tool.

\section{Data Acquisition}

We use a SP-150 potentiostat from Bio-logic(C) along with its commercial software EC-lab to perform the measurements. For each measurement, three sweeps in frequency are being measured, from $1 \mathrm{MHz}$ down to $0.1 \mathrm{~Hz}$. A sinusoidal voltage of $100 \mathrm{mV}$ peak-to-peak is applied. Five points per frequency decade, logarithmically spaced, are measured. For each data point the response to 10 consecutive sinusoids (but spaced out by $10 \%$ of the period duration) is accumulated and averaged.

\section{Supplementary Notes}

\section{Fitting procedure for Nyquist plots}

From the electrical model described in Figure 1c, the total impedance of the dielectric elastomer is

$$
Z_{d}=\frac{1}{j 2 \pi f C_{d}+\frac{1}{R_{d}+Z_{i}}}
$$

Where $C_{d}$ is the capacitance of the dielectric elastomer, $R_{d}$ its resistance due to ionic conductivity and $Z_{i}$ the interfacial impedance between the elastomer and the conductor. The interfacial impedance is often described in terms of charge a transfer resistance, an electrical double layer (capacitance) and a mass transfer impedance (Warburg's impedance model being the most widely used for electrolyte solutions) $)^{2}$. At high frequencies, the electrical double layer impedance as well as the mass transfer impedance are low. Only the charge transfer mechanism may contribute to a finite value of resistance at high frequencies. Because the elastomer thin films are thick, $R_{d}$ is large compare to mass transfer resistance. Thus, $Z_{i}$ becomes negligible compared to $R_{d}$ at low frequencies.

for $f \rightarrow 0 \mathrm{~Hz}$.

$$
Z_{d} \approx \frac{1}{j 2 \pi f C_{d}+\frac{1}{R_{d}}}
$$

The corresponding Nyquist plot is shown in Figure S4. Without interfacial impedance, the Nyquist plot is a semi-circle, which diameter is $R_{d}$. Two schematic curves are also shown to describe real Nyquist plots, where $Z_{i}$ dominates the elastomer's electrochemical impedance at low frequencies. In our experiments, we don't know exactly the nature of the interfacial impedance between the conductor and the elastomer, which is why we don't try to model it.

The fitting is done with the software EC-Lab. We first extrapolate the value of $C_{d}$ before soaking the sample in PBS solution. After soaking, we model the high-frequency part of the Nyquist plot (above $10 \mathrm{~Hz}$ ) as $R_{d}$ in parallel with $C_{d}$. The value of $C_{d}$ is known from the first measurement before soaking. From the value of $R_{d}$, we obtain the ionic conductivity $\sigma_{d}$ (using equation 3 of the main text).

\section{Molecular dynamics simulations 1. Overview}


The tasks of generating the polymer structures and assigning the OPLS 2005 force-field parameters are performed within the Materials Science suite from Schrodinger Inc. ${ }^{3-4}$, while all the molecular dynamics (MD) simulations are performed using the LAMMPS $\operatorname{code}^{5}$ and have periodic boundary conditions. For every polymer (PIB or SU-8) plus solute (either water or $\mathrm{NaCl}$ ) combination, five independently generated structures are created to calculate ensemble averages. Every structure is first equilibrated via a combination of energy minimization, compression/decompression, and heating/cooling stages following established protocols in the literature ${ }^{6-8}$, and then run for up to 70 ns while recording the position of the solute molecule over time to compute its diffusion coefficient via analysis of the mean-squared displacement (MSD).

2. Details on the structures.

To generate the polymer plus solute structures we first create a "low density" polymer matrix, in which a single water or $\mathrm{NaCl}$ molecule is added as a solute, Figure S6a. The low density is chosen to be roughly $50 \%$ of the experimental equilibrium density at $300 \mathrm{~K}$. For every polymer and solute combination, five independently generated structure are created to compute ensemble averages and uncertainties and to minimize the impact of specific polymer conformations. The structures are then brought close to equilibrium, Figure 1S6b, with an equilibration routine described in the next section. It is important to highlight that the chains are not cross-linked.

PIB. Every PIB structure comprises a total of 11584 atoms organized in 12 chains of 120 monomers each and terminated with methyl groups. At the end of the equilibration routine the cubic simulation box has a side length of roughly 47 Angstrom.

SU-8. Every SU-8 structure comprises a total of 11154 atoms organized in 3 chains of 61 monomers each and terminated with methyl groups. At the end of the equilibration routine the cubic simulation box has a side length of roughly 49 Angstrom.

3. Full equilibration breakdown.

\begin{tabular}{|c|c|c|c|c|}
\hline Stage & $\begin{array}{l}\text { Stage duration } \\
\text { [ns] }\end{array}$ & Total time [ns] & $\begin{array}{l}\text { Temperature } \\
{[\mathrm{K}]}\end{array}$ & Pressure $[\mathrm{MPa}]$ \\
\hline $\begin{array}{l}\text { Energy } \\
\text { minimization }\end{array}$ & - & - & - & - \\
\hline $\begin{array}{l}\text { NPT } \\
\text { (compression) }\end{array}$ & 0.10 & 0.10 & 353 & $0 \rightarrow 100$ \\
\hline $\begin{array}{l}\text { Energy } \\
\text { minimization }\end{array}$ & - & - & - & - \\
\hline $\begin{array}{l}\text { NPT } \\
\text { (compression) }\end{array}$ & 0.50 & 0.60 & 353 & $0 \rightarrow 400$ \\
\hline NPT & 0.40 & 1.00 & 353 & 400 \\
\hline $\begin{array}{l}\text { NPT } \\
\text { (decompression) }\end{array}$ & 0.60 & 1.60 & 353 & $400 \rightarrow 0.1$ \\
\hline NPT (heating) & 0.40 & 2.00 & $353 \rightarrow 453$ & 0.1 \\
\hline NPT (cooling) & 0.40 & 2.40 & $453 \rightarrow 353$ & 0.1 \\
\hline $\begin{array}{l}\text { NPT } \\
\text { (compression) }\end{array}$ & 0.30 & 3.00 & 353 & $0.1 \rightarrow 400$ \\
\hline $\begin{array}{l}\text { NPT } \\
\text { (decompression) }\end{array}$ & 0.30 & 3.00 & 353 & $400 \rightarrow 0.1$ \\
\hline NVT & 0.80 & 3.80 & 353 & - \\
\hline
\end{tabular}



NPT

Table S1. Stages used to equilibrate the polymer / diffusant initial structures. The 12 stages are sequentially run. During heating/cooling and compression/decompression stages, the initial/final temperature/pressure is indicated in the label as "Initial $\rightarrow$ Final". The change in temperature/pressure is performed at a constant rate over the entire duration of the stage.

\section{Density.}

See figure $\mathrm{S} 7$.

\section{Lossy transmission line bandwidth.}

The conductive interconnect insulated by a dielectric elastomer was modelled using a transmission line model ${ }^{9}$, for a stripline geometry (Figure 5e, f). Resistor $R_{c}$ is used to model the resistance per unit length of the conductor. $C_{d}$ represent the capacitance per unit length of the dielectric. $1 / R_{d}$ is the conductance per unit length of elastomer dielectric, representing the ionic conductivity of the material. In this part, we neglect the interfacial impedance for simplicity. These lumped electrical elements per unit length can be expressed as a function of geometry and materials parameters:

$$
\begin{aligned}
R_{c} & =\frac{1}{\sigma_{c}} \frac{1}{W H_{c}} \\
C_{d} & =2 \varepsilon_{d} \frac{W}{H_{d}} \\
\frac{1}{R_{d}} & =2 \sigma_{d} \frac{W}{H_{d}}
\end{aligned}
$$

The numerical pre-factor 2 comes from geometry: the conductor is insulated by the dielectric on both sides. In practice, $H_{c} \ll W$, thus we neglect electrical losses through the two other conductor - insulator interfaces.

Let's write $v(x, t)=\operatorname{Re}\left\{V(x) e^{j 2 \pi f t}\right\}$ and $i(x, t)=\operatorname{Re}\left\{I(x) e^{j 2 \pi f t}\right\}$. We derive the propagation equation in the frequency domain.

Applying Kirchhoff's voltage law and Ohm's law, the voltage change across the section $\Delta \mathrm{x}$ is:

$$
V(x+\Delta x)-V(x)=-R_{c} \Delta x I(x)
$$

Applying Kirchhoff's current law, the current change across the section $\Delta \mathrm{x}$ is:

$$
I(x+\Delta x)-I(x)=-\left(j 2 \pi f C_{d}+\frac{1}{R_{d}}\right) \Delta x V(x)
$$

For $\Delta \mathrm{x} \rightarrow 0$, we can rewrite equation (4) and (5) in terms of the first order derivative of the voltage and current:

$$
\begin{aligned}
& \frac{d V(x)}{d x}=-R_{C} \mathrm{I}(x) \\
& \frac{d I(x)}{d x}=-\left(j 2 \pi f C_{d}+\frac{1}{R_{d}}\right) V(x)
\end{aligned}
$$

By combining (3) and (4), we obtain the propagtion equation for a signal along the transmission line:

And

$$
\frac{d^{2} V(x)}{d x^{2}}=R_{c}\left(j 2 \pi f C_{d}+\frac{1}{R_{d}}\right) V(x)
$$

$$
\frac{d^{2} I(x)}{d x^{2}}=R_{c}\left(j 2 \pi f C_{d}+\frac{1}{R_{d}}\right) I(x)
$$

We define $\gamma$ where $\gamma^{2}=R_{c}\left(j 2 \pi f C_{d}+\frac{1}{R_{d}}\right)$ 


$$
\begin{aligned}
& \frac{d^{2} V(x)}{d x^{2}}=\gamma^{2} V(x) \\
& \frac{d^{2} I(x)}{d x^{2}}=\gamma^{2} I(x)
\end{aligned}
$$

The solution of these equations is:

$$
V(x)=V_{1} e^{-\gamma x}+V_{2} e^{+\gamma x}
$$

And

$$
I(x)=I_{1} e^{-\gamma x}+I_{2} e^{+\gamma x}
$$

Along a stripline interconnect of length $L$, the relative signal amplitude is $|V(L) / V(0)|$. Consider a forward-traveling wave, with traveling voltage $V_{1} e^{-\gamma x}$. The attenuation at a distance $L$ from the origin is $\left|\frac{V(L)}{V(0)}\right|=\left|e^{-\gamma L}\right|$, a function of the frequency $\omega$.

$$
\left|\frac{V(L)}{V(0)}\right|=\left|\exp \left(-\sqrt{R_{c}\left(2 \pi j f C_{d}+\frac{1}{R_{d}}\right)} L\right)\right|
$$

Which can be rewritten

$$
\left|\frac{V(L)}{V(0)}\right|=\left|\exp \left(-\sqrt{\frac{1}{f_{c}}\left(j f+f_{d}\right)}\right)\right|
$$

With $f_{c}=\frac{1}{4 \pi} \frac{H_{c} H_{d}}{L^{2}} \frac{\sigma_{c}}{\varepsilon_{d}}$ and $f_{d}=\frac{1}{2 \pi} \frac{\sigma_{d}}{\varepsilon_{d}}$. 


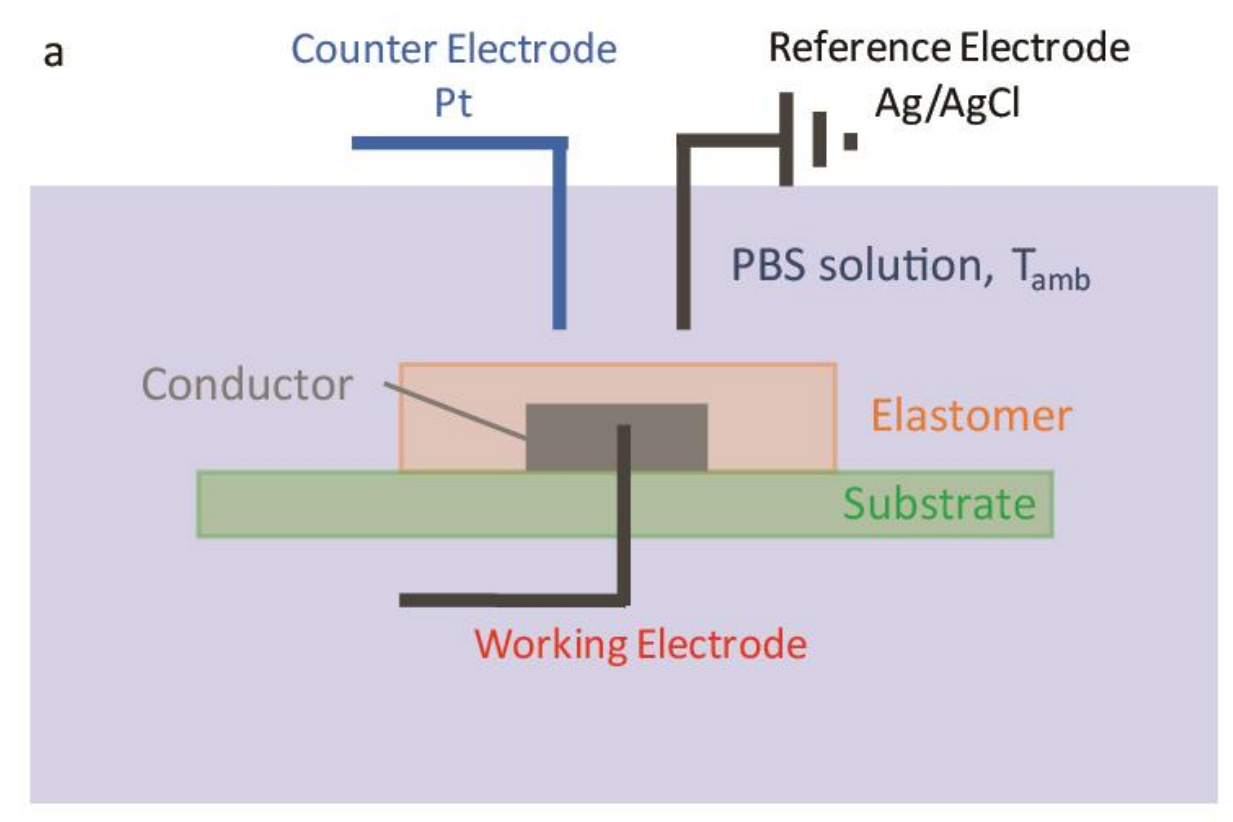

b

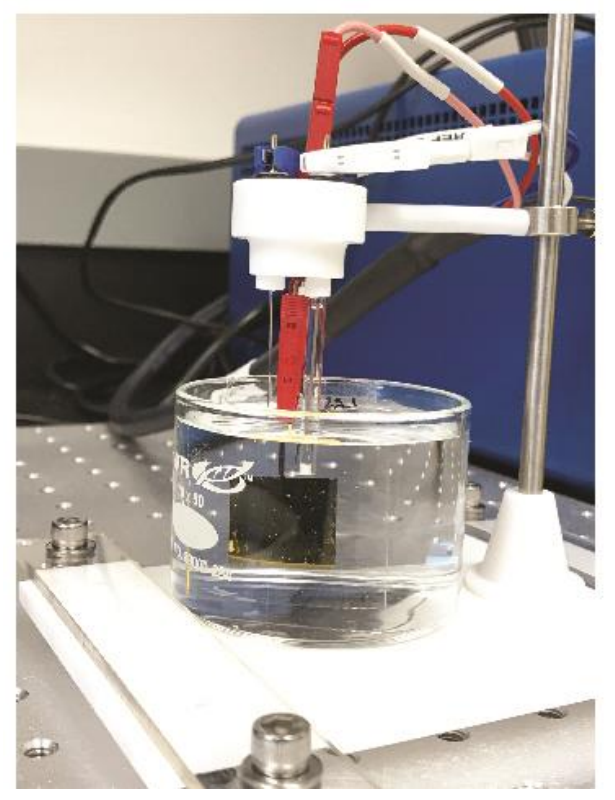

C

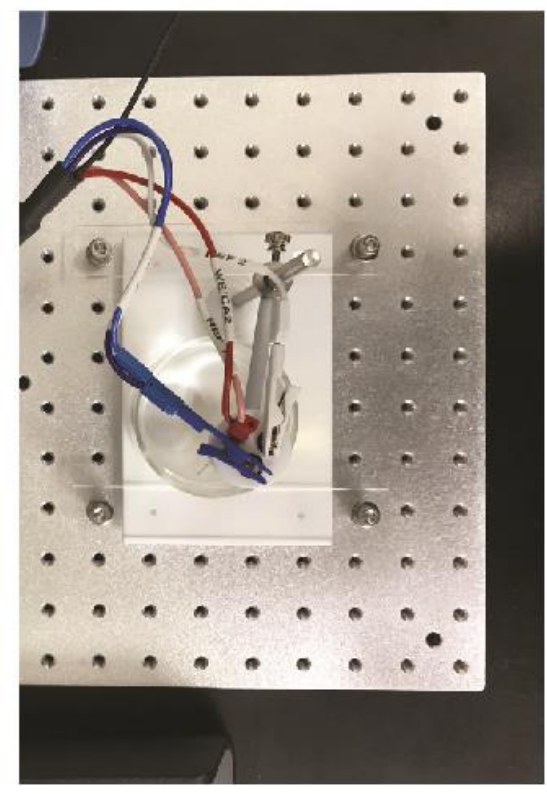

Figure S1. Schematics and images show the three-electrode set-up for EIS characterization. a, Schematic of the three-electrode setup for EIS characterization, consisting of 1) a working electrode connected to a conductor on one side of the dielectric layer, 2) a counter electrode (Platinum) and 3) a reference electrode (Silver/Silver Chloride). All three electrodes are immersed in 1x PBS solution. b-c, Photographic images of the setup mounted on a breadboard. 

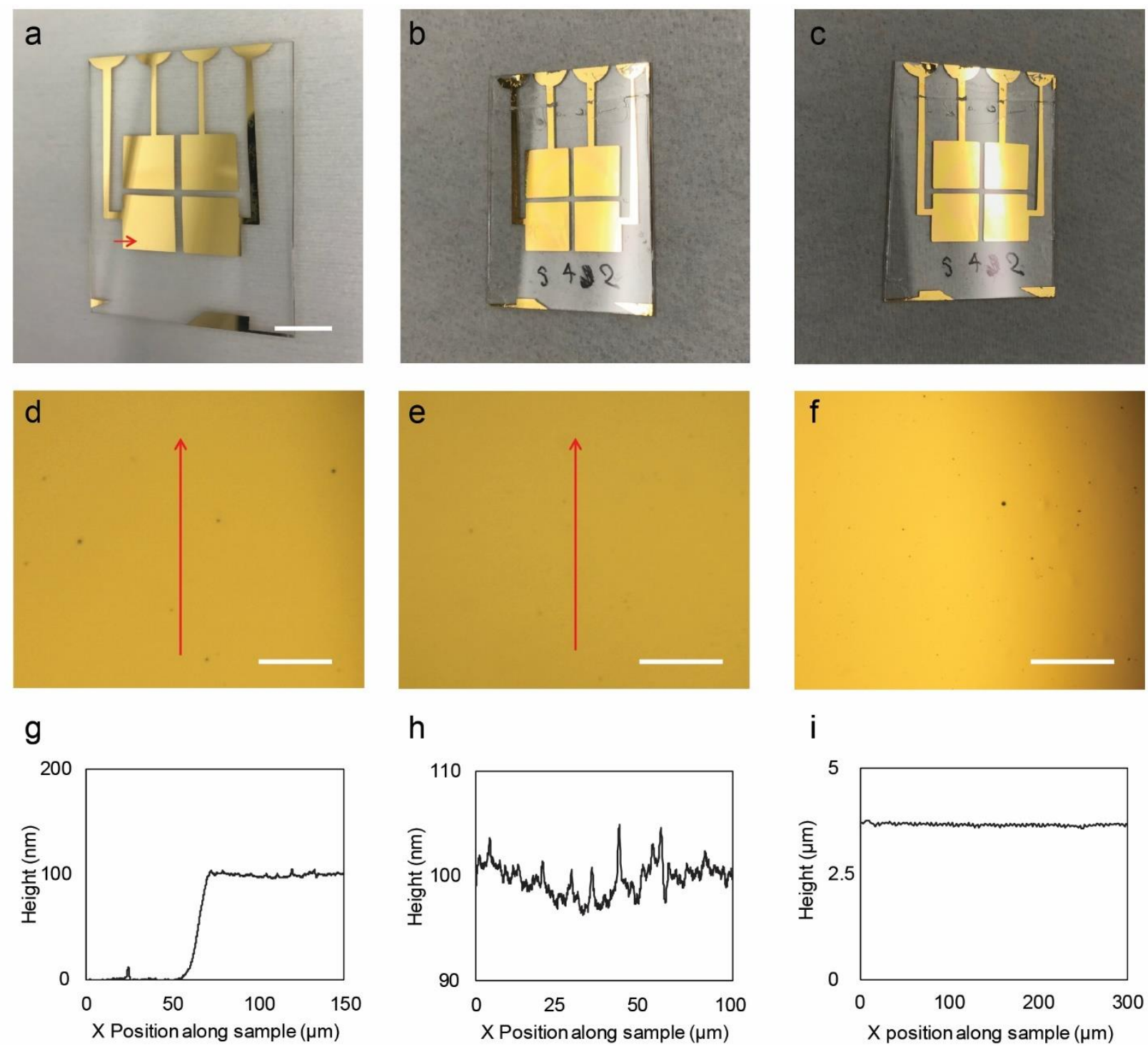

Figure S2. Imaging and profilometry characterizations show the integrity and uniformity of Au electrodes with elastomer coating. a, Photographic image shows four $\mathrm{Cr} / \mathrm{Au}$ electrodes patterned on a glass substrate for EIS characterization. (b, c) Photographic images show electrodes coated with dielectric elastomer (e.g., SEBS in these images) before (b) and after (c) soaking in 1x PBS solution. No defects in the coating are observed by visual inspection before and after soaking. (d-f) Bright-field (BF) microscopic images correspond to the samples presented in (a-c) respectively. g, Contact profilometry of the gold electrode deposited on a glass slide, along the red arrow shown in (a). h, Contact profilometry of the gold electrode patterned on glass slide, along the red arrow shown in (d). i, Contact profilometry of the gold electrode coated with SEBS, along the red arrow shown in (e). Scale bars: $1 \mathrm{~cm}(\mathrm{a}-\mathrm{c}), 10 \mu \mathrm{m}(\mathrm{d}, \mathrm{e}), 40 \mu \mathrm{m}$ (f). 

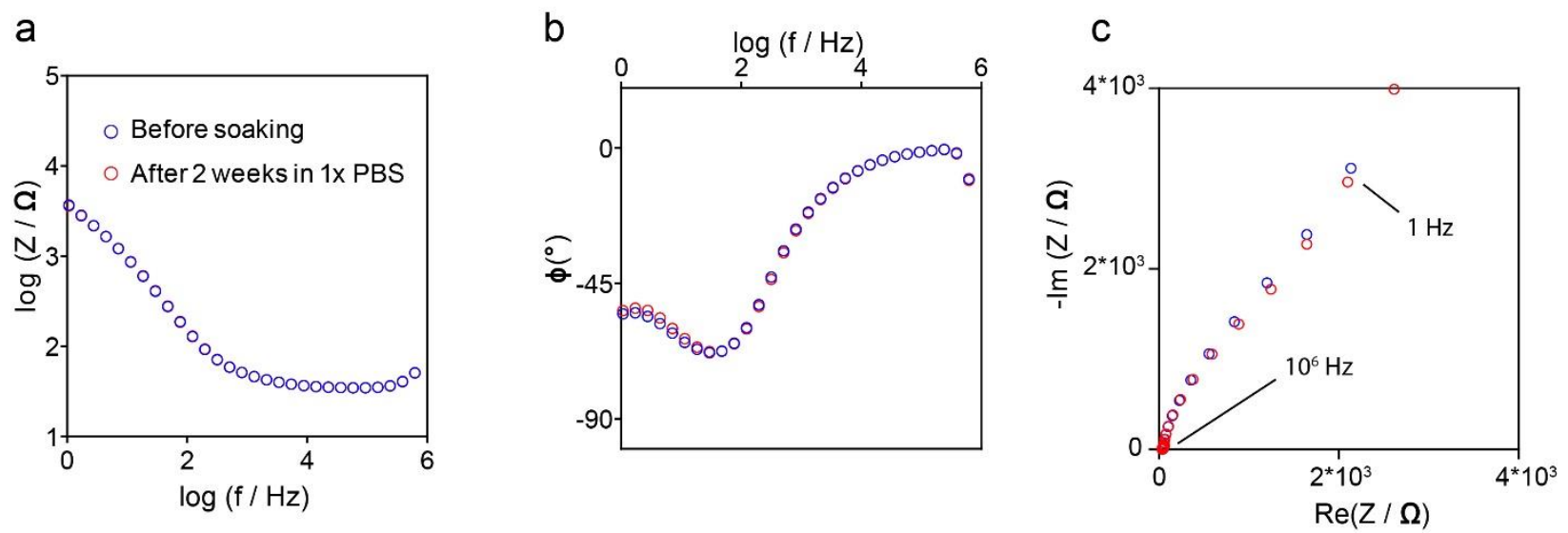

Figure S3. EIS shows the stability of the Cr/Au electrodes in PBS. (a, b) Impedance (a) and phase (b) plots of $1 \mathrm{~cm}^{2} \mathrm{Cr} / \mathrm{Au}$ electrode (5/100 nm thickness) before and after 2-week soaking in 1x PBS. (c) Nyquist plot of the same electrode. 


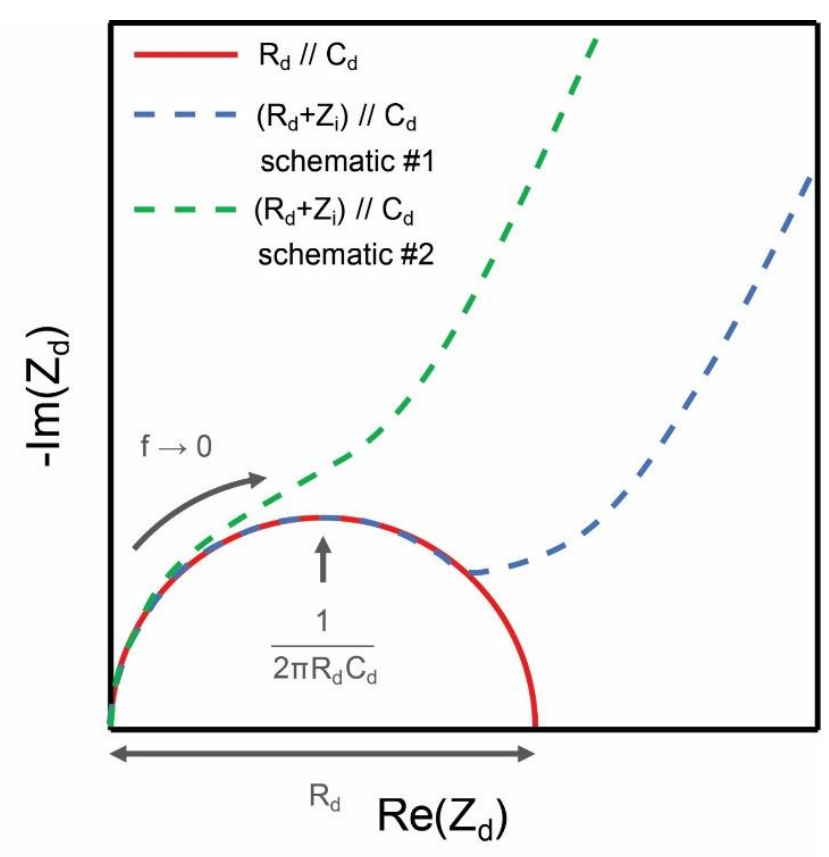

Figure S4. Schematics show how interfacial impedance affects the shape of the Nyquist plot The $R_{d} / / C_{d}$ plot is derived from equation 2. Deviation from the ideal $R_{d} / / C_{d}$ plot can happen at different frequencies and have different scalings with the frequency, as illustrated by schematics $\# 1$ and \#2. This plot is from Bard et al. ${ }^{2}$ 
a
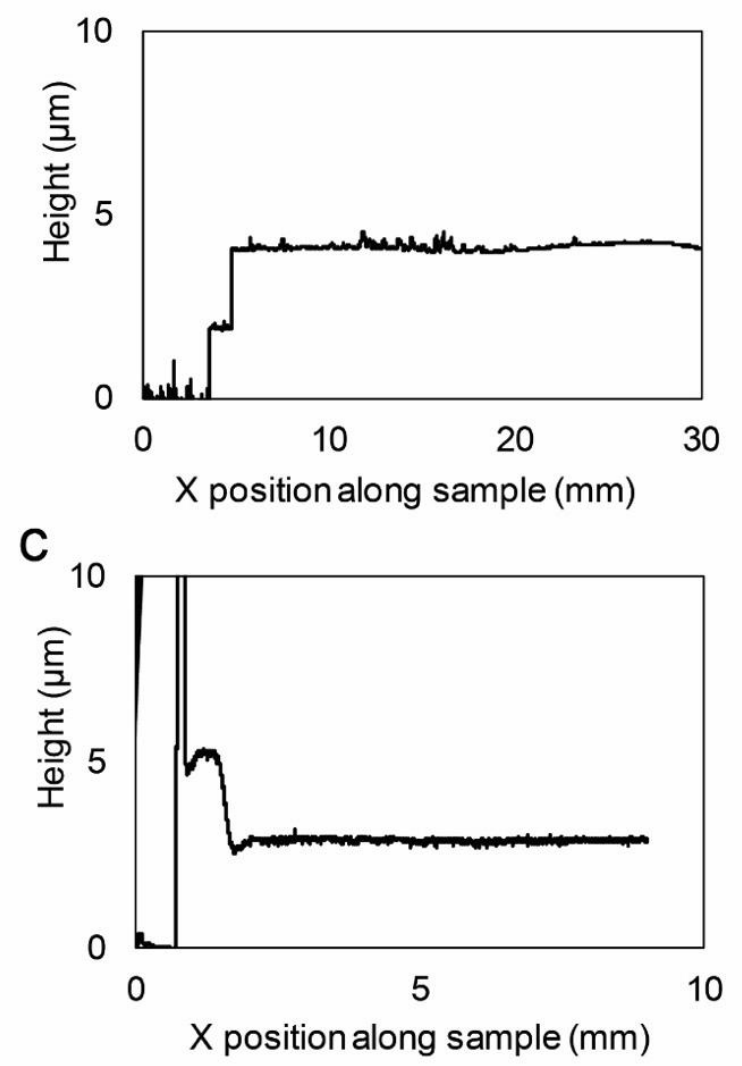

b
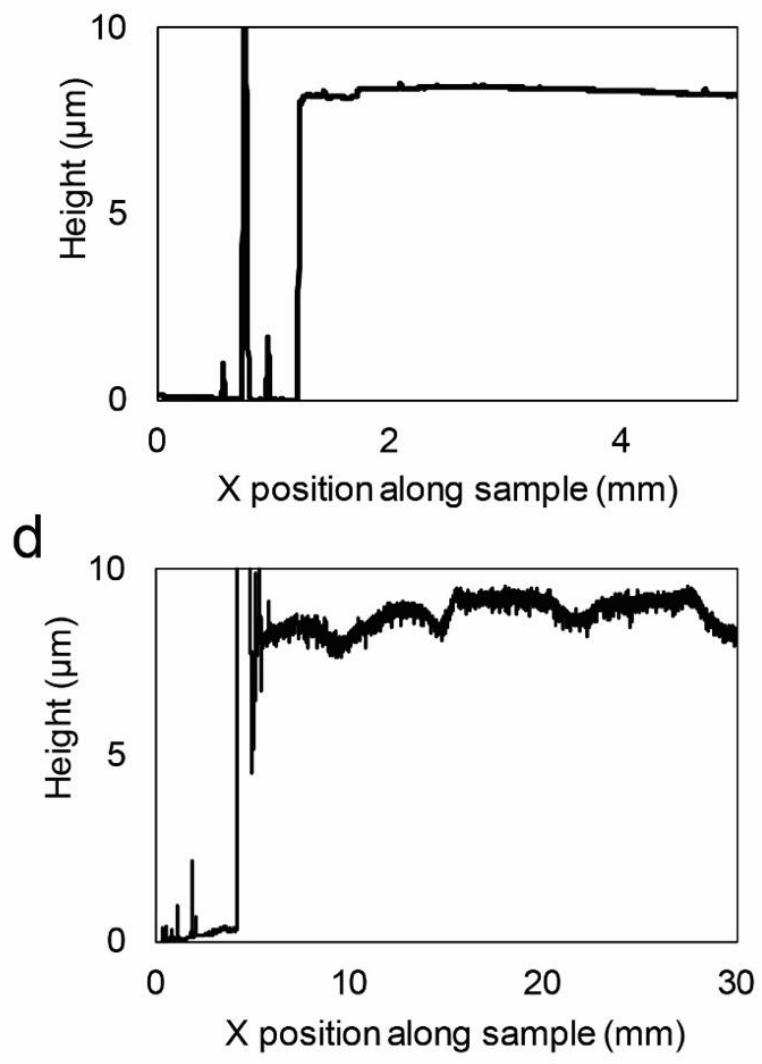

Figure S5. Contact profilometry measures the thickness of polymers spin coated on gold electrode. a, Thickness of two layers of SU-8 2002 spincoated on the gold electrode. (b-d), Thickness of single layers of PDMS (b), SEBS (c) and PIB (d) spincoated on gold electrodes. 
a

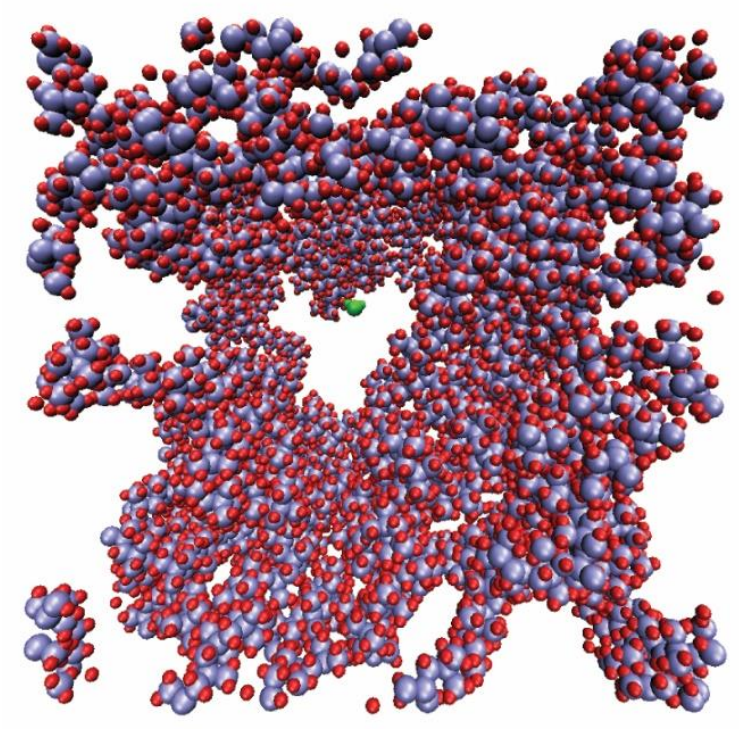

b

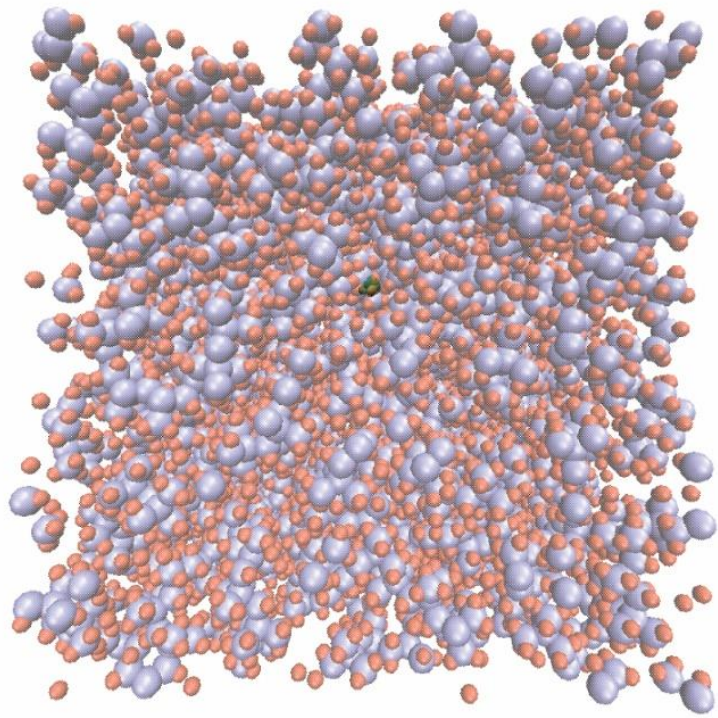

From low density ( $50 \%$ of expected density @ 300K) to equilibrium density @ 300K

Figure S6. Molecular Dynamics simulation generated using LAMMPS shows the water interaction with PIB. 12 polymer chains each made of 120 monomers are displayed. (a-b), Water/PIB structure before (a) and after (b) the equilibration routine. The water molecule is highlighted in green color. 


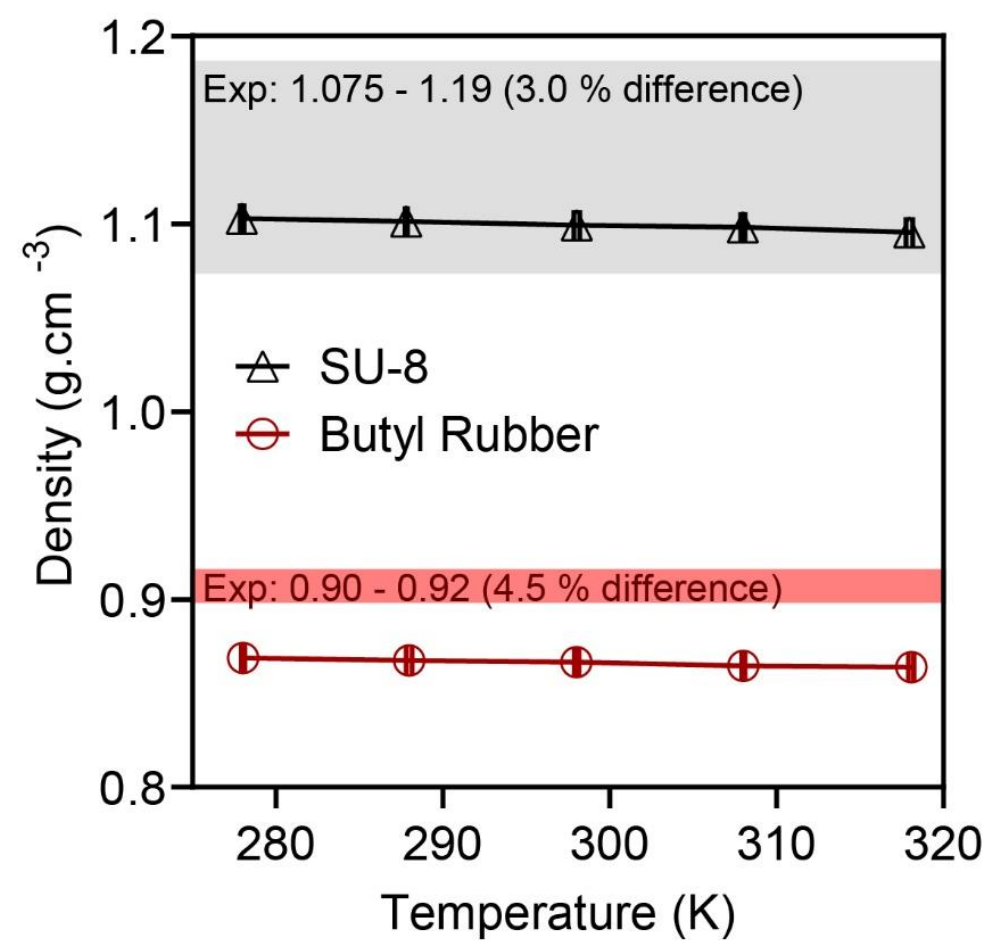

Figure S7. Computed densities of PIB and SU-8 at different temperatures are compared to experimental values. PIB structures simulated by MD simulation have slightly lower densities than experimental values reported in the literature (shaded areas), while SU-8 structures have similar densities ${ }^{6-8,10-12}$. 
a

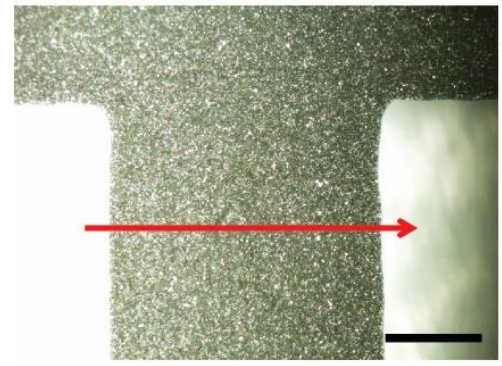

d

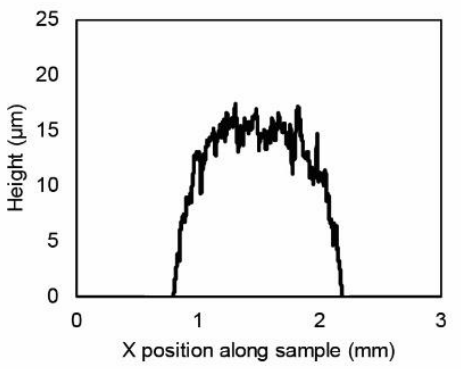

b

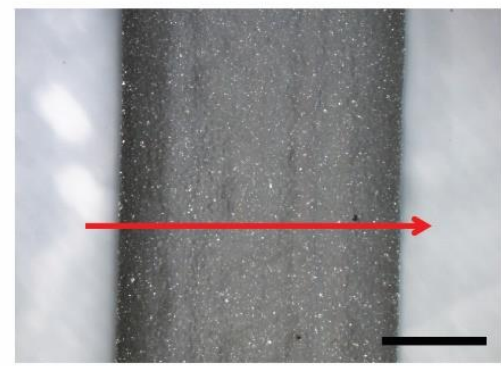

e

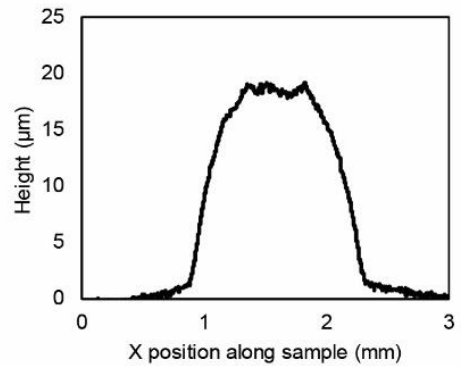

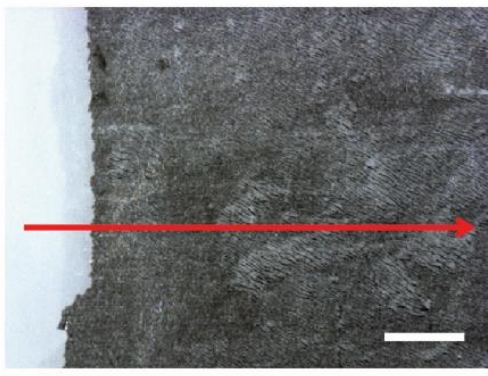

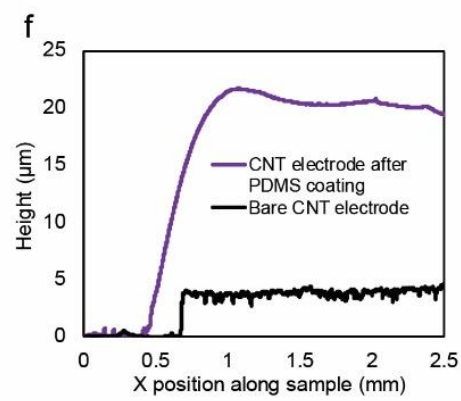

$\mathrm{g}$

$\mathrm{h}$
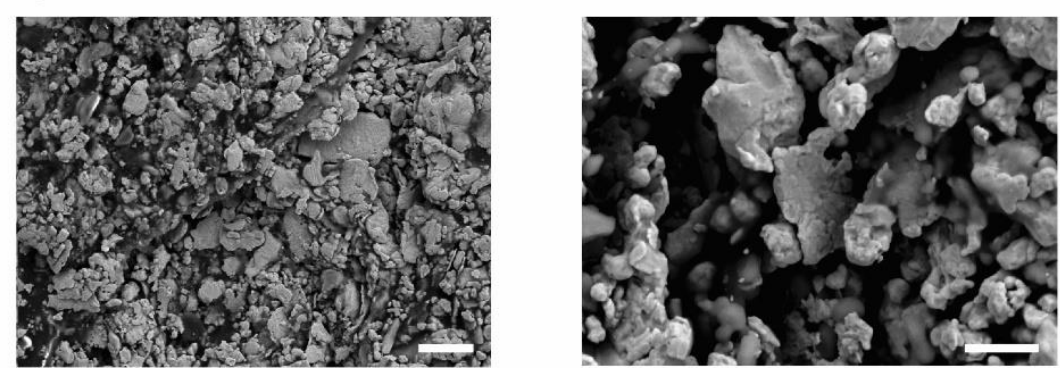

Figure S8. Imaging and profilometry characterizations show the integrity of stretchable electrodes with elastomer. a, Ag nanoparticles - polymer composite interconnect cured on a glass slide. b, The same interconnect coated with SEBS spin coated on top. c, CNT electrode coated with two layers of PDMS spin coated on top of each other. d, Height profile along the red arrow shown in (a). e, Height profile along the red arrow shown in (b). f, Height profile along the red arrow shown in (c). (g, h) SEM images of the Silver nanoparticles - polymer composite ink after curing. Silver flakes are in grey. Scale bars, $500 \mu \mathrm{m}(\mathrm{a}, \mathrm{b}), 250 \mu \mathrm{m}(\mathrm{c}), 3 \mu \mathrm{m}(\mathrm{g}), 1 \mu \mathrm{m}(\mathrm{h})$, 
a

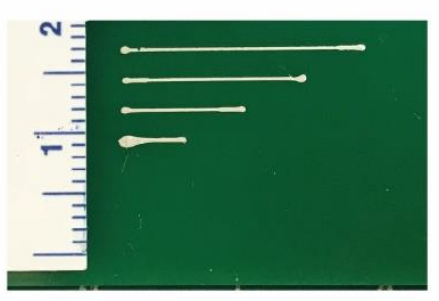

b

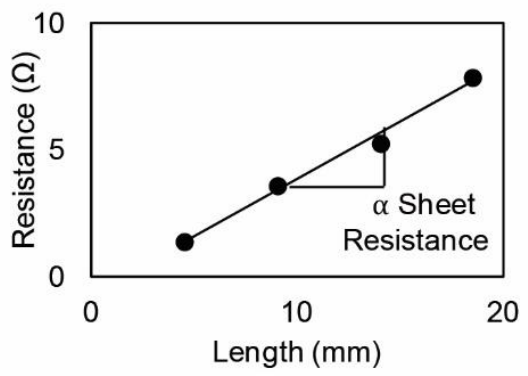

C

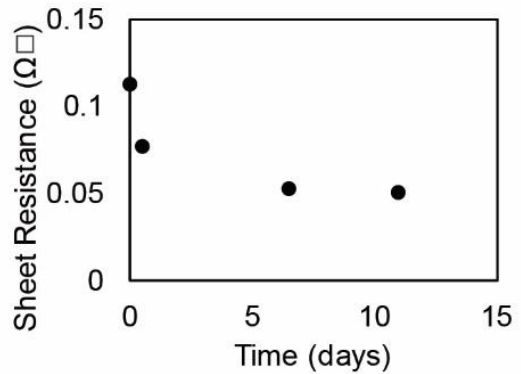

Figure S9. Conductance measurement shows the stability of Ag nanoparticle electrodes in 1xPBS. a, Conductive traces of PE873 cured on a PCB board (green). b. The resistance of the trace is linearly proportional to its length, and the slope of the curve is proportional to the sheet resistance of the ink. c, The sheet resistance of PE873 decreases slightly over time an reaches a stable value after a few days when immersed in 1x PBS. 

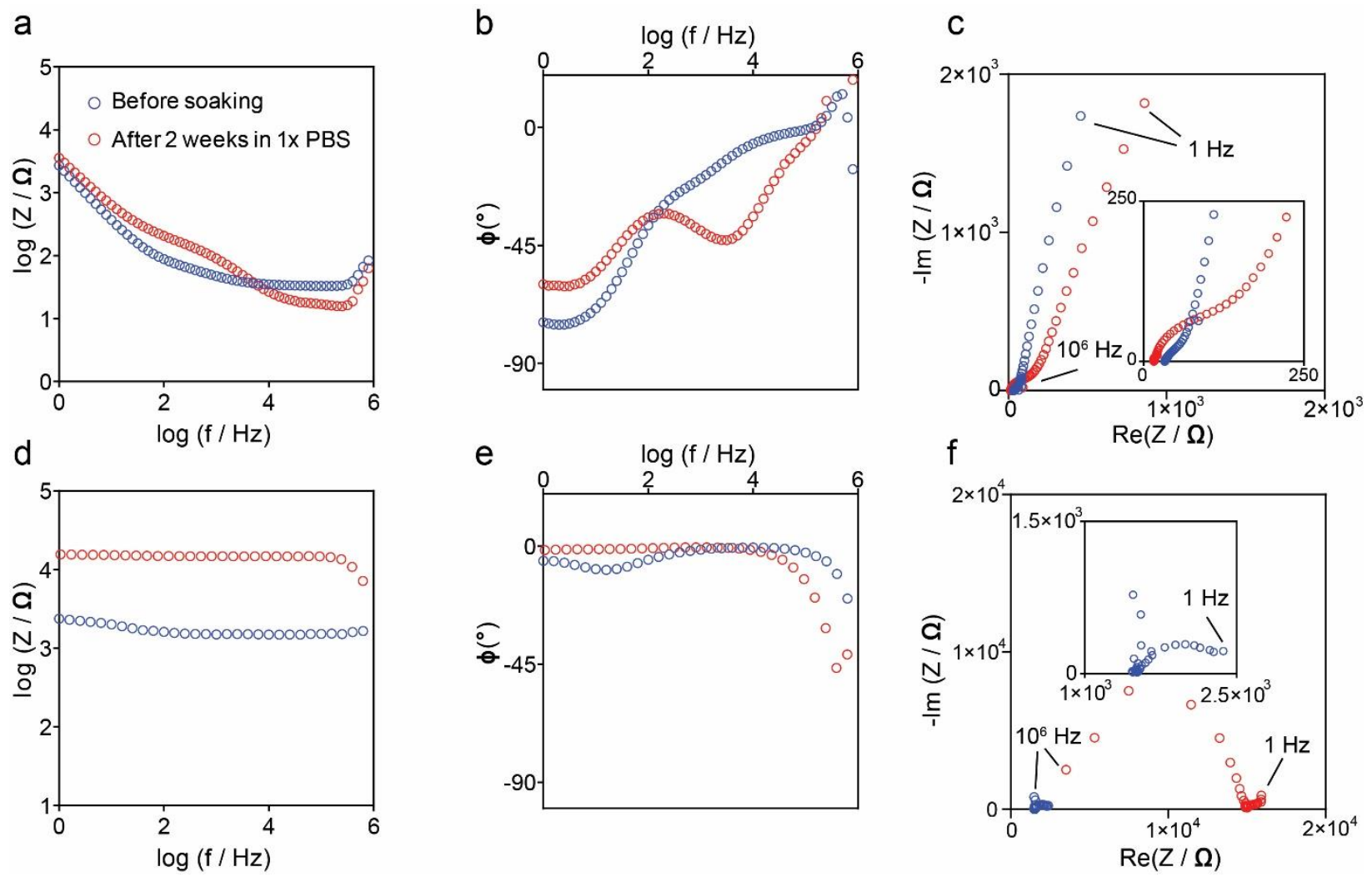

Figure S10. EIS shows the stability of the stretchable electrodes in PBS. (a, b) impedance (a) and phase (b) plots of $1 \mathrm{~cm}^{2}$ siler nanoparticles - polymer composite electrode (12 $\mu \mathrm{m}$ thickness). (c) Nyquist plot of the same electrode. (d, e) impedance (d) and phase (e) plots of $1 \mathrm{~cm}^{2} \mathrm{CNT}$ electrode $(20 \mu \mathrm{m}$ thickness). (f) Nyquist plot of the same electrode. 


\section{References}

1. Zhao, H.; Hussain, A. M.; Duduta, M.; Vogt, D. M.; Wood, R. J.; Clarke, D. R., Compact Dielectric Elastomer Linear Actuators. Adv. Funct. Mater. 2018, 28 (42), 1804328.

2. Bard, A. J.; Faulkner, L. R.; Leddy, J.; Zoski, C. G., Electrochemical Methods: Fundamentals and Applications. Wiley: New York, 1980; Vol. 2.

3. Banks, J. L.; Beard, H. S.; Cao, Y.; Cho, A. E.; Damm, W.; Farid, R.; Felts, A. K.; Halgren, T. A.; Mainz, D. T.; Maple, J. R., Integrated Modeling Program, Applied Chemical Theory (IMPACT). J. Comput. Chem. 2005, 26 (16), 1752-1780.

4. Jorgensen, W. L.; Maxwell, D. S.; Tirado-Rives, J., Development and Testing of the OPLS All-atom Force Field on Conformational Energetics and Properties of Organic Liquids. J. Am. Chem. Soc. 1996, 118 (45), 11225-11236.

5. Plimpton, S., Fast Parallel Algorithms for Short-range Molecular Dynamics. J. Comput. Phys. 1995, 117 (1), 1-19.

6. Kucukpinar, E.; Doruker, P., Molecular Simulations of Gas Transport in Nitrile Rubber and Styrene Butadiene Rubber. Polymer 2006, 47 (22), 7835-7845.

7. Molinari, N.; Mailoa, J. P.; Kozinsky, B., Effect of Salt Concentration on Ion Clustering and Transport in Polymer Solid Electrolytes: A Molecular Dynamics Study of PEO-LiTFSI. Chem. Mater. 2018, 30 (18), 6298-6306.

8. Molinari, N.; Khawaja, M.; Sutton, A.; Mostofi, A., Molecular Model for HNBR with Tunable Cross-link Density. J. Phys. Chem. B 2016, 120 (49), 12700-12707.

9. Chipman, R. A., Theory and Problems of Transmission Lines. McGraw-Hill Book Company: New York, 1968.

10. http://www.mit.edu/ 6.777/matprops/su-8.htm.

11. http://www-mdp.eng.cam.ac.uk/web/library/enginfo/cueddatabooks/materials.pdf.

12. http://microchem.com/pdf/SU-8\%203000\%20Data\%20Sheet.pdf. 